\title{
In honor of Professor Kiyokatsu Jinno
}

\author{
Stephen A. Wise ${ }^{1}$
}

Published online: 14 October 2016

(C) Springer-Verlag Berlin Heidelberg 2016

At the end of July 2016, Professor Kiyokatsu Jinno retired as an editor of Analytical and Bioanalytical Chemistry (ABC). He was one of the six founding editors when the first issue of the journal appeared in January 2002.

Kiyokatsu Jinno (Fig. 1) received his BA (1968), MS degree (1970), and $\mathrm{PhD}$ (1973) all from Nagoya University in Nagoya, Japan, working with Professor Daido Ishii, a prominent Japanese separation scientist. Professor Jinno's doctorate degree, however, was in engineering with research in non-destructive elemental analysis using neutron activation analysis. He then spent the next five years as a research scientist in the Integrated Circuit Laboratory at the Toshiba Research and Development Center working on the development of the dry plasma etching process and electron beam lithographic techniques for large-scale integrated circuits, which he described as "an active and interesting field" [1]. In 1978 he was invited to become an associate professor in the School of Materials Science at Toyohashi University of Technology (TUT), a new national technical university just opening at the time of his arrival. At TUT he had been expected to work in the area of radiochemistry using an accelerator that was planned to be built on campus. Unfortunately, the accelerator never appeared, and Professor Jinno was left to find a new area of research. Following the advice of his mentor, Professor Ishii, he chose micro-column liquid chromatography (micro-LC) as his area of research. Professor Jinno

Stephen A. Wise

stephen.wise@nih.gov

1 National Institutes of Health (NIH), 6100 Executive Blvd, Room 3B01, MSC 7517, Bethesda, MD 20892-7517, USA remained at TUT until his retirement in 2014 as Full Professor and Vice President of the University.

Professor Jinno's decision to move from activation analysis and elemental analysis to the emerging area of LC was monumental, and he soon became an internationally recognized leader in the field of separation science and related sample preparation techniques. His areas of research during his career span the broad scope of separation science including retention mechanisms and molecular recognition in LC, design of novel stationary phases, development of capillary separation techniques, miniaturized sample preparation techniques and their combination with liquid-phase separations, hyphenated techniques in chromatography and spectroscopy, and the use of computers in separation sciences. He has contributed to a wide range of separation techniques, beyond his primary focus in LC, including micro-LC, capillary electrophoresis, supercritical fluid chromatography (SFC) and extraction (SFE), electrochromatography, gas chromatography, gel permeation chromatography, high-performance thin-layer chromatography, chiral chromatography, solid-phase extraction (SPE), and solid-phase microextraction (SPME).

Professor Jinno has been a prolific author with over 310 original research papers, 35 review articles, 39 book chapters, and four books (as author and editor). The exceptional number of review articles, book chapters, and books illustrates the broad range of his contributions. He has presented over 90 plenary and invited lectures at international symposia on chromatography and analytical chemistry.

In 2001 a book titled A Century of Separation Science was published highlighting the careers and experiences of many of the founding leaders in the field of separation science. In Professor Jinno's contribution, "My Life in Separation Science: Study of Separation Mechanisms" [1], he states that "his contribution to the development of separation science can be divided into at least two main categories: hyphenated 


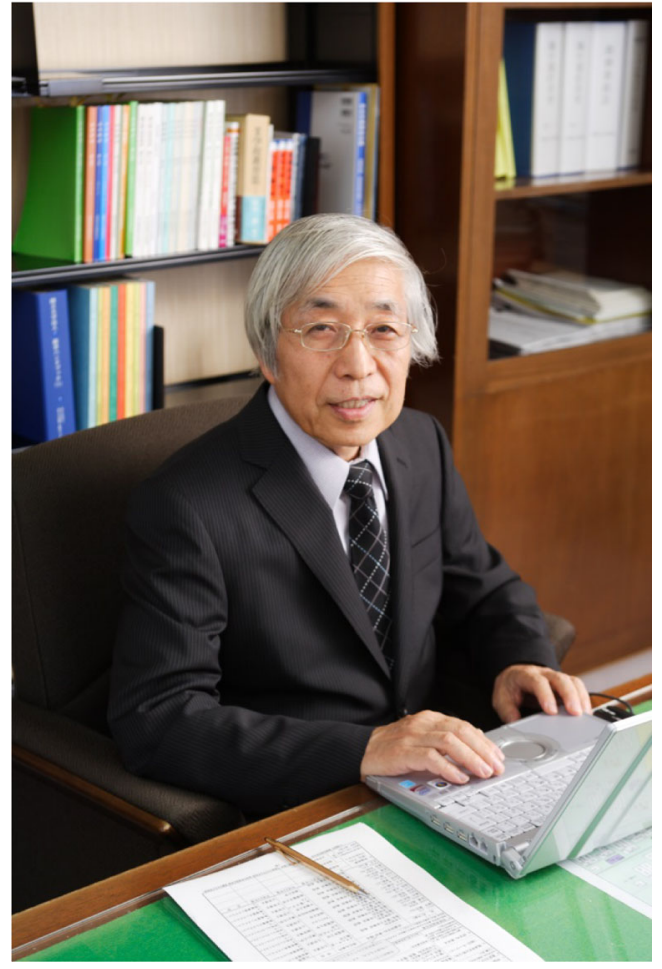

Fig. 1 Professor Kiyokatsu Jinno

techniques in liquid phase separations and the study of retention mechanisms in liquid phase separations". He then discusses his contributions to the study retention mechanisms in LC.

Professor Jinno's investigations related to molecular recognition and retention mechanisms in LC, particularly using polycyclic aromatic hydrocarbons (PAHs) as test solutes, are substantial with over 100 publications that have contributed significantly to the understanding of retention mechanisms in LC, and specifically to the separation of PAHs on a variety of stationary phases. His studies on the correlation of LC retention behavior and molecular parameters (e.g., shape, non-planarity, and polarizability) are numerous and significant. This body of work represents his most highly cited papers. In 1996 he was the editor and a contributor to the book Chromatographic Separations Based on Molecular Recognition [2]. This book provided a comprehensive review and interpretation of research in molecular recognition and shape selective separations in LC. He was one of the first researchers to pursue chromatographic separations of fullerenes after the discovery of buckyballs in 1985, and he produced numerous (ca. 35) publications on this subject over the next decade. Professor Jinno collaborated with numerous other researchers in separation science. One of his most productive collaborations, resulting in over 20 publications, was with John C. Fetzer at Chevron Research working with high molecular mass PAHs and investigating their LC separation on a different stationary phases.
As part of Professor Jinno's efforts to understand retention mechanisms in LC, he developed and evaluated numerous new materials for and approaches to creating novel stationary phases for LC, e.g., mono-, di-, and tri-phenyl; $\mathrm{C}_{30}$; cholesteryl; dicoronylene; $\mathrm{C}_{60}$ and $\mathrm{C}_{70}$ fullerenes; fluorinated alkanes; liquid crystal; $\mathrm{Cu}$-phthalocyanine; cyclodextrins; polyvinyl alcohol; cellulose acetate fibers; chitosan; ceramic clay; tri-tert-butylphenoxy; and chiral phases. Most of these investigations focused on chromatographic characterization of the novel phases; however, some studies also included chemical characterization of the stationary phases from different preparation approaches using techniques such as NMR. One objective in understanding chromatographic retention mechanisms and behavior is to be able to predict chromatographic retention for identification when an authentic standard is not available. Professor Jinno pursued this goal by implementing computer-assisted retention prediction systems for PAHs and other chemical groups of interest. His research group was one of the earliest to embrace the use of computer systems in the chemistry laboratory to assist in chromatographic analysis.

Another research area in which he has had significant impact is sample preparation approaches, and particularly the miniaturization and coupling of sample preparation techniques (e.g., SFE, SPE, and SPME) to liquid-phase separation techniques. He was also one of the first chromatographers to recognize the potential benefits and impact of micro-LC techniques including packed micro columns, packed capillary tubes, and open tubular columns. Professor Jinno's group led the way as innovators in coupling micro-LC and SFC techniques with non-traditional (at the time) spectroscopic detectors for chromatography such as Fourier transform infrared, inductively coupled plasma, and atomic emission spectroscopy.

Professor Jinno's research was not just directed towards understanding separations, but also to applying a variety of separation techniques in environmental analyses (e.g., PAHs in diesel particulate material, pesticides/insecticides, estrogens in water) and clinical/ food analyses (e.g., uric acid, drugs, vitamins, carotenoids, and ginsenosides).

For his significant contributions to chromatography and related separation techniques, Professor Jinno has been recognized with prestigious international awards including the Tokai Industrial Chemistry Award (1986), the Russian Tswett Medal (1996), University of Helsinki Medal (1998), Golay Award (1999), Award of the Chromatographic Sciences (2000), Society Award from the Japanese Society for Analytical Chemistry (2003), and the Latin-American Congress on Chromatography (COLACRO) Medal (2012). Professor Jinno was one of the first generation of researchers in the field of modern liquid chromatography and related separation techniques, and he is 
recognized among those considered as the founding leaders in LC and separation science. He is truly an exceptional chromatographer!

A great part of his legacy are the 71 Masters and $\mathrm{PhD}$ students whom he mentored and graduated during his career at Toyohashi University of Technology. One of his students, Yoshihiro Saito, authored editorials in Chromatographia honoring him on the occasion of his 60th birthday (2005) [3] and in $A B C$ honoring him on the occasion of his 70th birthday (2015) [4].

I first recognized the name of Kiyokatsu Jinno early in the 1980s, since we both had interests in LC separations of PAHs and investigations of unique molecular recognition and shape selectivity for PAHs in reversed-phase LC. However, my first significant time with him was in October 1989 as I participated in an international chromatography symposium in Tokyo and then travel to Toyohashi to present a lecture at TUT at his invitation. He was a very gracious host, and we spent an enjoyable dinner and evening at his home leaving me with very favorable memories of my first visit to Japan.

Prior to becoming an editor of $A B C$, he served as an editor for Chromatographia and on the editorial boards of several journals including Analytical Chemistry, Journal of Chromatography, and Journal of Chromatographic Science. For $A B C$ his most significant contribution has been his dedication to the journal and his efforts to make it a premier journal in analytical and bioanalytical chemistry. As a recognized leader in separation science, he worked hard to bring highquality separation science research papers to $A B C$. He served as a guest editor for Topical Collections $[5,6]$ inviting his colleagues to contribute to $A B C$. Until recently, he was the only $A B C$ editor in the Asia-Pacific region, and he represented $A B C$ well and stimulated the journal's growth and recognition in this region, particularly in Japan.

From the beginning of $A B C$, Professor Jinno has been a "quiet" giant among the editors, always providing thoughtful comments and insight during the editors' discussions. We will miss his quiet presence at our meetings. From all the editors and the editorial office at $A B C$, we thank Professor Kiyokatsu Jinno for his service to the journal and to the separation science and analytical/bioanalytical chemistry communities in general. We wish him the best in his retirement from the journal.

\section{References}

1. Jinno K. My life in separation sciences: study of separation mechanisms. In: Issaq HJ, editor. A century of separation science. New York: Dekker; 2001. p. 253-63.

2. Jinno K, editor. Chromatographic separations based on molecular recognition. New York: Wiley; 1996.

3. Saito Y. Editorial: 60th birthday of Professor Kiyokatsu Jinno. Chromatographia. 2005;61:323.

4. Saito Y. Editorial: 70th birthday of Professor Kiyokatsu Jinno. Anal Bioanal Chem. 2015;407:2651.

5. Jinno K. Modern sample preparation techniques. Anal Bioanal Chem. 2002;373:1-2.

6. Wise SA, Jinno K, Takeuchi T. Advances in separation science. Anal Bioanal Chem. 2005;382:533-4. 